\title{
MEASURABLE PARAMETRIZATIONS OF SETS IN PRODUCT SPACES
}

\author{
BY
}

V. V. SRIVATSA

\begin{abstract}
Various parametrization theorems are proved. In particular the following is shown: Let $B$ be a Borel subset of $I \times I$ (where $I=[0,1]$ ) with uncountable vertical sections. Let $\Sigma \cup N$ be the discrete (topological) union of $\Sigma$, the space of irrationals, and $N$, the set of natural numbers with discrete topology. Then there is a map $f: I \times(\Sigma \cup N) \rightarrow I$ measurable with respect to the product of the analytic $\sigma$-field on $I$ (that is, the smallest $\sigma$-field on $I$ containing the analytic sets) and the Borel $\sigma$-field on $\Sigma \cup N$ such that $f(t, \cdot): \Sigma \cup N \rightarrow I$ is a one-one continuous map of $\Sigma \cup N$ onto $\{x:(t, x) \in B\}$ for each $t \in T$. This answers a question of Cenzer and Mauldin.
\end{abstract}

0. Introduction. Let $(T, \mathscr{T})$ be a measurable space and $X$ a Polish space. Suppose $B \in \mathfrak{R} \otimes \mathfrak{B}_{X}$, where $\mathfrak{B}_{X}$ is the Borel $\sigma$-field of $X$, such that each vertical section of $B$ is uncountable. In this article we consider the following kind of "parametrization" problem: Find a map $f: T \times Y \rightarrow X$, where $Y=\Sigma$, the space of irrationals, or $Y=\Sigma \biguplus N$, the discrete (topological) union of $\Sigma$ and $N$, the set of natural numbers with discrete topology, such that $f(t, \cdot)$ is a one-one continuous function on $Y$ onto $B^{t}$ and $f$ has suitable measurability properties. We then call $f$ a one-one Carathéodory map and $Y$ the parametrizing space. Several authors have considered this kind of problem and an extensive bibliography is to be found in Wagner [14].

We begin, in $\S 2$, with parametrizations of certain kinds of measurable $G_{\delta}$-valued multifunctions. The basic result is a "uniform" version of Mazurkiewicz's theorem that a dense $G_{\delta}$ subset of a zero-dimensional Polish space whose complement is also dense is a homeomorph of $\Sigma$, that is, we obtain here a Borel measurable $f$ : $T \times \Sigma \rightarrow X$ such that $f(t, \cdot)$ is a homeomorphism onto $B^{t}$ for each $t \in T$. We use this technique to obtain one-one Carathéodory representations for measurable dense-in-itself $G_{\delta}$-valued multifunctions. These results on $G_{\delta}$-valued multifunctions are used in the sequel. We might add that R. D. Mauldin and H. Sarbadhikari have independently obtained similar results [8].

In $\$ 3$ we solve a question posed by Cenzer and Mauldin [3] on the parametrization of Borel subsets $B$ of $I \times I$ with uncountable sections where $I$ is the unit interval. Wesley [15] had earlier taken the first step in this problem when he showed, using forcing techniques, that there is an $\mathcal{L}_{2}$-measurable map $f: I \times I \rightarrow I$, where $\mathcal{L}_{2}$ is the Lebesgue $\sigma$-field on $I \times I$, such that $f(t, \cdot)$ is a Borel isomorphism on $I$ onto $B^{t}$ for

Received by the editors May 20, 1980.

1980 Mathematics Subject Classification. Primary 04A15; Secondary 03E15, 28A05, 28A20, 54H05.

Key words and phrases. Parametrizations, selections. 
each $t$. Cenzer and Mauldin [3] obtained a more "descriptive" result when they showed, without any use of metamathematical methods, that there is an $S(I \times I)$ measurable map $f$ with the above property (where $S(X)$ denotes the class of $C$-sets in $X$ ). However, a well-known result of von Neumann and Yankov states that such a $B$ always has a $\mathscr{B}(\mathscr{Q}(I))$-measurable selector, where $\mathscr{B}(\mathscr{Q}(I))$ is the smallest $\sigma$-field on $I$ containing all the analytic sets. Cenzer and Mauldin therefore asked whether $B$ can be so parametrized that each of the selectors for $B$ induced by the map $f$ is $\mathscr{B}(\mathscr{Q}(I))$-measurable. We show that this is indeed possible. In fact we obtain a one-one Carathéodory map $f$ which is $\mathscr{B}(\mathscr{Q}(I)) \otimes \mathfrak{B}_{r}$-measurable. It should be pointed out, however, that in [3] Cenzer and Mauldin had also proved that given an analytic set $A \subset I \times I$ there is a $\mathscr{B}(\mathscr{Q}(I \times I))$-measurable map $f: I \times C \rightarrow I$ such that for each $t \in I, f(t, \cdot)$ is a homeomorphism of $C$ into $B^{t}$. Our methods are then used to improve results of Ioffe [4] and Bourgain [2]. Their result is the following. Let $(T, \Re, \mu)$ be a complete measure space, $\mu$ being a $\sigma$-finite measure. Then any $B \in \Re \otimes \Re_{X}$ with uncountable vertical sections is induced by a one-one, $\mathbb{R} \otimes$ $\Re_{\Sigma \cup N}$-measurable Carathéodory map $f: T \times(\Sigma \cup N) \rightarrow X$. We establish an abstract version of our theorem and conclude that the result of Ioffe and Bourgain holds when $\Re$ is any $\sigma$-field closed under operation $(\mathscr{Q})$.

In $\S 4$, the concluding section, we show that if $B$ is assumed to have condensed vertical sections, then in some of our results the parametrizing space $\Sigma \cup N$ can be replaced by $\Sigma$. These correspond to uniform versions of the fact that a set is condensed Borel if and only if it is a one-one continuous image of $\Sigma$.

The author is grateful to Professor Ashok Maitra for the many helpful discussions he had with him.

1. Definitions and notation. We denote by $N$ the set of all natural numbers and Seq will denote the set of all finite sequences of natural numbers, including the empty sequence $e$. For $k \in N, S_{k}$ will be the set of all elements of Seq of length $k$. For $s \in$ Seq, $|s|$ will denote the length of $s$ and if $i<|s|$ is a natural number, $s_{i}$ will denote the $(i+1)$ st coordinate of $s$. Thus, for $s \in S_{k}$, we write $s=\left(s_{0}, s_{1}, \ldots, s_{k-1}\right)$ or $s=\left\langle s_{0}, s_{1}, \ldots, s_{k-1}\right\rangle$. We use $l(s)$ to denote the last coordinate of $s$. For $i \leqslant|s|$, $s \uparrow i$ denotes $\left(s_{0}, s_{1}, \ldots, s_{i-1}\right)$ and for $s, t \in$ Seq, we use $s \bar{t}$ to denote the catenation of $s$ and $t$. If $n \in N$, sn denotes the catenation of $s$ and $\langle n\rangle$. For $s, t \in$ Seq, we write $s \prec t$ if $t$ extends $s$. We put $\Sigma=N^{N}$. Endowed with the product of discrete topologies on $N, \Sigma$ becomes a homeomorph of the space of irrationals. For $\sigma \in \Sigma$ and $i \in N, \sigma_{i}$ will denote the $i$ th coordinate of $\sigma$ and $\sigma i i$ will denote the finite sequence $\left(\sigma_{0}, \sigma_{1}, \ldots, \sigma_{i-1}\right)$; here if $i=0, \sigma_{i} i$ will just be the empty sequence. If $s \in S$ then the set $\left\{\sigma \in \Sigma: \sigma_{i}=s_{i}\right.$ for $\left.i<|s|\right\}$ will be denoted by $\Sigma(s)$. Finally, $\Sigma \cup N$ denotes the discrete (topological) union of $\Sigma$ and $N, N$ being given the discrete topology.

Let $T$ and $X$ be nonempty sets and $\mathscr{Q} \subseteq \mathcal{P}(T)$, the power set of $T$. A multifunction $F: T \rightarrow X$ is a function whose domain is $T$ and whose values are subsets of $X$. If nothing explicit is stated the values of $F$ will be nonempty. For $E \subseteq X$, we denote by $F^{-1}(E)$ the set $\{t \in T: F(t) \cap E \neq \varnothing\}$. By $\operatorname{Gr}(F)$ we mean the set $\{(t, x) \in T \times X$ : $x \in F(t)\}$, and call it the graph of $f$. A function $f: T \rightarrow X$ is called a selector for $F$ if 
$f(t) \in F(t), t \in T$. If $\mathcal{Q}$ and $\mathscr{B}$ are $\sigma$-fields on $T$ and $X$, respectively, then $\mathfrak{A} \otimes \mathfrak{B}$ will denote the product $\sigma$-field on $T \times X$. If $\mathscr{P}$ is a family of subsets of $T, \sigma(\mathscr{P})$ will denote the smallest $\sigma$-field containing $P$.

Now suppose $T$ is a nonempty set and $Q \subseteq \mathcal{P}(T)$. Let $X$ and $Y$ be metric spaces. A multifunction $F: T \rightarrow X$ is called $Q$-measurable if $F^{-1}(V)$ is in $Q$ for every open set $V \subseteq X$. Similarly, a point map $f: T \rightarrow X$ is $\mathscr{Q}$-measurable if $f^{-1}(V) \in \mathbb{Q}$ for every $V$ open in $X$. If $S$ is a nonempty set and $\mathscr{B} \subseteq P(S)$, then $f: T \rightarrow S$ is ( $Q, \mathscr{B}$ )-measurable if $f^{-1}(B) \in \mathbb{Q}$ for every $B \in \mathscr{B}$. A map $f: T \times Y \rightarrow X$ is called an $\mathcal{Q}$-measurable Carathéodory map if $f(t, \cdot): Y \rightarrow X$ is continuous for each $t \in T$, and $f(\cdot, y): T \rightarrow X$ is $Q$-measurable for each $y \in Y$. If $f: T \times Y \rightarrow X$ is a Carathéodory map then $\hat{f}$ : $T \times Y \rightarrow T \times X$ denotes the map defined by $\hat{f}(t, y)=(t, f(t, y))$, and is called the canonical map induced by $f$. Observe that if $Y$ is separable and $f$ is an $Q$-measurable Carathéodory map then $f$ and $\hat{f}$ are both $\left(Q \otimes \mathscr{B}_{Y}\right)$-measurable.

By $\Re_{X}$ we will mean the Borel $\sigma$-field of $X$. Now let $X$ be Polish. Then $\mathscr{B}(\mathscr{Q}(X))$ denotes the analytic $\sigma$-field on $X$, that is, the smallest $\sigma$-field on $X$ containing the analytic subsets of $X$. If $Y \subseteq X, \Re(Q(Y))$ denotes the trace of $\mathscr{B}(\mathscr{Q}(X))$ on $Y$.

Now suppose $\Re$ is a countably generated $\sigma$-field on $T$ generated by $\left\{M_{n}\right\}_{n \geqslant 1}$. By the characteristic function of the sequence $\left\{M_{n}\right\}$ we mean the function $f: T \rightarrow[0,1]$ given by

$$
f(t)=\sum_{n=1}^{\infty} \frac{2}{3^{n}} \cdot I_{M_{n}}(t)
$$

where $I_{M_{n}}$ is the indicator function of the set $M_{n}$. Let $S=f(T)$. Then, as is well known, $f$ is a bimeasurable function (that is, a measurable function that carries measurable sets to measurable sets) between $\mathscr{N}$ and $\mathscr{B}_{S}$.

For $E \subseteq X, \operatorname{cl}(E)$ will denote the closure of $E$ and $\delta(E)$ the diameter of $E$.

For terminology not defined we refer the reader to Kuratowski [6].

2. Homeomorphic Carathéodory representations for $G_{\delta}$-valued multifunctions. In this section we will obtain homeomorphic Carathéodory representations for certain types of measurable $G_{\delta}$-valued multifunctions taking values in a zero-dimensional space. A typical result proved is a uniform version of Mazurkiewicz's theorem that a dense $G_{\delta}$-subset of a zero-dimensional space whose complement is also dense is a homeomorph of the space of irrationals (see [6]). Our method is to carry out the proof for the "single-section" case uniformly.

For the rest of this section $T$ and $X$ will be Polish spaces, $X$ being, moreover, zero-dimensional. We fix a countably generated sub- $\sigma$-field $Q$ of the Borel o-field on $T$, $\mathscr{B}_{T}$. We also fix an Q-measurable $G_{\delta}$-valued multifunction $F: T \rightarrow X$. The graph of $F$ will be denoted by $G$, and we will assume that $G \in \mathbb{Q} \otimes \mathscr{B}_{X}$. Apart from the assumption on the dimension of $X$, this is the set-up in Srivastava [12] where the basic selection theorem for $G_{\delta}$-valued multifunctions has been proved.

We begin by stating three well-known lemmas. The first two are proved in [12] and the third is a result of Blackwell [1]. 
Lemma 2.1. Let $T, X$ be Polish spaces and let $Q$ be a countably generated sub- $\sigma$-field of $\mathscr{B}_{T}$. Suppose $B \in \mathbb{Q} \otimes \mathscr{B}_{X}$ and $B^{t}$ is $a G_{\delta}$ in $X$ for each $t \in T$. Then there exist sets $B_{n} \in \mathbb{Q} \otimes \mathscr{B}_{X}$ such that $B_{n} \supseteq B_{n+1}, B_{n}^{t}$ is open in $X$ for each $t \in T$ and $n \geqslant 1$ and $B=\bigcap_{n \geqslant 1} B_{n}$.

Lemma 2.2. Let $(T, \mathscr{Q})$ and $(X, \mathfrak{B})$ be measurable spaces. Let $\mathbb{Q}$ be atomic and $B \in \mathbb{Q} \otimes \mathscr{B}$. Then $B^{t}=B^{t^{\prime}}$ holds for $t$ and $t^{\prime}$ belonging to the same atom of $\mathbb{Q}$.

Lemma 2.3. Let $T$ be Polish and $\mathbb{Q}$ a countably generated sub- $\sigma$-field of $\mathscr{B}_{T}$. Then $A \in \mathbb{Q}$ iff $A \in \mathscr{B}_{T}$ and $A$ is a union of atoms of $\mathbb{Q}$.

Invoking Lemma 2.1 we write $G=\cap_{n \geqslant 0} G_{n}$ where $G_{n} \supset G_{n+1}, G_{n} \in \mathbb{Q} \otimes \mathscr{B}_{X}$, and $G_{n}^{t}$ is open for each $n \geqslant 0, t \in T$. Also $\left\{r_{0}, r_{1}, \ldots\right\}$ will be a fixed dense subset of $X, d$ a fixed metric for $X$, and $\{V(n)\}_{n \geqslant 0}$ a fixed nonempty clopen base for $X$. Denote the projection function on $T \times X$ to $T$ by $\pi_{T}$. We will now prove several lemmas.

Lemma 2.4. Let $T, X$ be as above. Assume further that $X$ is compact. Suppose $B \subseteq T \times X, B \in \mathbb{Q} \otimes \mathscr{B}_{X}$ and $B^{t}$ is open for each $t \in T$. Then $\left\{t \in T: B^{t}\right.$ is not closed $\} \in \mathbb{Q}$.

Proof. It follows from Lemma 2.3 and a result of Kunugui and Novikov [5] that

$$
\pi_{T}((T \times X)-B) \in \mathbb{Q}
$$

and that $(T \times X)-B$ is the graph of an $\mathscr{Q} \cap \pi_{T}((T \times X)-B)$-measurable, compact valued multifunction $H$ on $\pi_{T}((T \times X)-B)$. Now use the facts that $B^{t}$ is open and $X$ is compact to verify that

$\left\{t \in T: B^{t}\right.$ is not closed $\}$

$$
=\left\{t \in \pi_{T}((T \times X)-B):(\forall n)(\exists m)\left(r_{m} \in B^{t} \text { and } d\left(r_{m}, H(t)\right)<1 /(n+1)\right)\right\} .
$$

It is now easy to see that the set on the left belongs to $\mathcal{Q}$.

Lemma 2.5. Let $T, X$ be as in Lemma 2.4. Suppose $G^{t}$ is not closed for each $t \in T$. Let $\varepsilon>0$. Then there exist $\mathbb{Q}$-measurable maps $p(\cdot, n): T \rightarrow \omega$ for each $n \geqslant 0$ such that:

(i) $\delta(V(p(t, n)))<\varepsilon, t \in T, n \geqslant 0$.

(ii) $V(p(t, n))-\bigcup_{m<n} V(p(t, m)) \neq \varnothing$.

(iii) $G^{t} \subseteq \cup_{n \geqslant 0} V(p(t, n)) \subseteq G_{0}^{t}$.

Proof. Let $T_{n}=\left\{t \in T: G_{n}^{t}\right.$ is not closed $\}, n \geqslant 0$. By Lemma 2.4 each $T_{n} \in \mathcal{Q}$. As $G^{t}=\bigcap_{n \geqslant 0} G_{n}^{t}$ and $G^{t}$ is not closed for each $t \in T$, we see that for each $t$ there is $n \in \omega$ such that $G_{n}^{t}$ is not closed. Thus $\cup_{n \geqslant 0} T_{n}=T$. Disjointify the $T_{n}$ 's: set $S_{0}^{\prime}=T_{0}, S_{n}^{\prime}=T_{n}-\cup_{i<n} T_{i}, n \geqslant 1$. Then

$$
\bigcup_{n \geq 0} S_{n}^{\prime}=\bigcup_{n \geq 0} T_{n}=T, \quad S_{n}^{\prime} \subseteq T_{n}, \quad S_{n}^{\prime} \in \mathbb{Q},
$$

and the $S_{n}^{\prime}$ 's are pairwise disjoint. Let $C=\cup_{n \geqslant 0}\left(\left(S_{n}^{\prime} \times X\right) \cap G_{n}\right) . C \in \mathbb{Q} \otimes \Re_{X}$, $G \subseteq C \subseteq G_{0}$, and $C$ has open, nonclosed vertical sections. 
Define, for $n \geqslant 0$,

$$
\begin{aligned}
R_{n} & =\left\{t \in T: V(n) \subseteq C^{t}\right\} \quad \text { if } \delta(V(n))<\varepsilon, \\
& =\varnothing \quad \text { otherwise. }
\end{aligned}
$$

Notice that if $\delta(V(n))<\varepsilon$, then $R_{n}$ is the complement of the projection of a Borel subset of $T \times X$ with compact vertical sections. It follows that $R_{n} \in \mathscr{B}_{T}$. Moreover, $R_{n}$ is a union of $\mathcal{Q}$-atoms. It follows from Lemma 2.3 that $R_{n} \in \mathbb{Q}$. Now define, by induction on $n$ :

$$
\begin{aligned}
p(t, 0)=m & \text { if } m \text { is the first natural number } l \text { such that } t \in R_{l} . \\
p(t, n+1)=m & \text { if } m \text { is the first natural number } l>p(t, n) \\
& \text { such that } V(l)-\bigcup_{i \leqslant n} V(p(t, i)) \neq \varnothing \text { and } t \in R_{l} .
\end{aligned}
$$

Observe that as $C^{t}$ is not closed for every $t$, the $p(t, n)$ 's are well defined on the whole of $T$. For, if not, there would be $t$ and $n$ such that $\cup_{m \leqslant n} V(p(t, m))=C^{t}$, which is impossible as the $V(n)$ 's are clopen sets. One easily checks that the $p(t, n)$ 's are $Q$-measurable functions such that $\cup_{n \geq 0} V(p(t, n))=C^{t}$. They, therefore, satisfy the conditions in the lemma.

LemMA 2.6. Let the hypotheses of Lemma 2.5 be in force. Then there exist Q-measurable maps $p(\cdot, n): T \rightarrow \omega$ for each $n \geqslant 0$ such that:

(i) $\delta(V(p(t, n)))<\varepsilon, t \in T, n \geqslant 0$.

(ii) $\left(V(p(t, n))-\bigcup_{m<n} V(p(t, m))\right) \cap G^{t} \neq \varnothing$.

(iii) $G^{t} \subseteq \cup_{n \geqslant 0} V(p(t, n)) \subset G_{0}^{t}$.

Proof. Apply Lemma 2.5 to get maps $q_{0}^{\prime}(t, n), n \geqslant 0$, such that $\delta\left(V\left(q_{0}^{\prime}(t, n)\right)\right)<\varepsilon$ for $t \in T, n \geqslant 0$;

$$
V\left(q_{0}^{\prime}(t, n)\right)-\bigcup_{m<n} V\left(q_{0}^{\prime}(t, m)\right) \neq \varnothing
$$

and

$$
G^{t} \subseteq \bigcup V\left(q_{0}^{\prime}(t, n)\right) \subseteq G_{0}^{t}
$$

Let

$$
T_{n}=\left\{t \in T:\left(V\left(q_{0}^{\prime}(t, n)\right)-\bigcup_{m<n} V\left(q_{0}^{\prime}(t, m)\right)\right) \cap G^{t} \neq \varnothing\right\} .
$$

As $G$ is the graph of an $Q$-measurable multifunction and the functions $q_{0}^{\prime}(t, n)$ are $Q$-measurable, it follows that $T_{n} \in \mathbb{Q}$ for $n \geqslant 0$. For the purposes of this proof we will make a temporary definition by putting $V(\infty)=\varnothing$. Define, by induction on $n$, functions $q_{0}(t, n)$ as follows: Put

$$
\begin{aligned}
q_{0}(t, 0) & =q_{0}^{\prime}(t, k), \quad \text { where } k \text { is the first integer } l \text { such that } t \in T_{l} ; \\
q_{0}(t, n+1) & =q_{0}^{\prime}(t, k), \quad \text { if } k \text { is the }(n+2) \text { th integer } l \\
& \text { such that } t \in T_{l}, \text { if there is one such; } \\
& =\infty \quad \text { otherwise. }
\end{aligned}
$$

Then $q_{0}(t, n), n \geqslant 0$, are $Q$-measurable extended natural number-valued maps. 
Moreover,

$$
G^{t} \subset \bigcup_{n \geq 0} V\left(q_{0}(t, n)\right) \subseteq G_{0}^{t}
$$

For each $k \geqslant 0$, apply the above argument to $T, X, G,\left\{G_{n}, n \geqslant k\right\}$. Then, for each $k \geqslant 0$, we will obtain $Q$-measurable extended natural number-valued maps $q_{k}(t, n)$, $n \geqslant 0$, with the following properties:

(a) $\delta\left(V\left(q_{k}(t, n)\right)\right)<\varepsilon$ for $t \in T, n \geqslant 0$.

(b) $G^{t} \subseteq \bigcup_{n \geqslant 0} V\left(q_{k}(t, n)\right) \subseteq G_{k}^{t}$.

$$
q_{k}(t, n)<\infty \Rightarrow\left(V\left(q_{k}(t, n)\right)-\bigcup_{m<n} V\left(q_{k}(t, m)\right)\right) \cap G^{t} \neq \varnothing .
$$

Define $C_{k} \subseteq T \times X$ by

$$
(t, x) \in C_{k} \leftrightarrow x \in \bigcup_{n \geqslant 0} V\left(q_{k}(t, n)\right) .
$$

As the $q_{k}(t, n)$ 's are $Q$-measurable maps, $C_{k} \in \mathbb{Q} \otimes \Re_{X}$ for $k \geqslant 0$. Moreover, $C_{k}^{t}$ is open, $k \geqslant 0, t \in T$, and $\bigcap_{k \geqslant 0} C_{k}=G$. Now, by assumption $G^{t}$ is not closed for each $t$. Consequently, for each $t \in T$ there is $k \geqslant 0$ such that $C_{k}^{t}$ is not closed. Put $S_{n}^{\prime}=\left\{t \in T: C_{n}^{t}\right.$ is not closed $\}, n \geqslant 0$. By Lemma $2.4, S_{n}^{\prime} \in \mathbb{Q}$ for $n \geqslant 0$. Disjointify the $S_{n}^{\prime}$ 's: Set $R_{0}=S_{0}^{\prime}, R_{n}=S_{n}^{\prime}-\cup_{i<n} S_{i}^{\prime}, n \geqslant 1$. Then $\cup_{n \geqslant 0} R_{n}=T$, the $R_{n}$ 's are pairwise disjoint, $C_{n}^{t}$ is not closed for $t \in R_{n}$, and $R_{n} \in \mathbb{Q}$ for $n \geqslant 1$. Define $C \subseteq T \times X$ by

$$
C=\bigcup_{n \geqslant 0}\left(\left(R_{n} \times X\right) \cap C_{n}\right) .
$$

Then $C \in \mathbb{Q} \otimes \mathscr{B}_{X}, G \subseteq C \subseteq G_{0}$ and $C$ has open, nonclosed sections. For $t \in R_{k}$, $C^{t}=C_{k}^{t}$. As $C^{t}$ is not closed, $C_{k}^{t}=\cup_{n \geqslant 0} V\left(q_{k}(t, n)\right)$ is not closed. Since the $V(n)$ 's are from a clopen base, it follows that on $R_{k}, q_{k}(t, n)<\infty$ for every $n \geqslant 0$. To complete the proof we now need only put $p(t, n)=q_{k}(t, n)$ if $t \in R_{k}$.

Lemma 2.7. Let $T$ and $X$ be as in Lemma 2.4. Assume that for each clopen $V$ in $X$ such that $G^{t} \cap V \neq \varnothing$, we have $G^{t} \cap V$ is not closed. Then there is a system $\{p(t, s)$ : $s \in \mathrm{Seq}\}$ of $Q$-measurable maps on $T$ into $\omega$ such that:

(i) $s \in S_{k}, k \geqslant 1 \Rightarrow \delta(V(p(t, s)))<\frac{1}{k}$ for $t \in T$.

(ii) For $s \in S_{k}$,

$$
(V(p(t, s)))-\bigcup\left\{V(p(t,(s \uparrow(k-1)) i)): i<s_{k-1}\right\} \cap G^{t} \neq \varnothing .
$$

(iii) For $s \in S_{k}, n \geqslant 0$,

$$
V(p(t, s n)) \subseteq V(p(t, s))-\bigcup\left\{V(p(t,(s \uparrow(k-1)) i)): i<s_{k-1}\right\} .
$$

$$
G^{t} \subseteq \bigcup\left\{V(p(t, s)): s \in S_{k}\right\} \subseteq G_{k-1}^{t}, \quad t \in T, \quad k \geqslant 1 .
$$

Proof. We will define such a system by induction on $|s|$. Put $p(t, e)=$ least $n$ such that $V(n)=X$ (we assume here for convenience that $X$ is in $\{V(n)\}_{n \geqslant 0}$ ). For $|s|=1$, we obtain maps $p(t, n)$ as in Lemma 2.6 with $\varepsilon=1$. Suppose $p(t, s)$ has been defined for $s \in S_{k}$. Fix $s \in S_{k}$. We have to define $p(t, s n)$ for $n \geqslant 0$. Let 
$j=s_{k-1}$. For $d \in S_{j+1}$, define

$$
T(d)=\left\{t \in T: p(t,(s \uparrow(k-1)) 0)=d_{0}, \ldots, p\left(t,(s \uparrow(k-1)) s_{k-1}\right)=d_{j}\right\} .
$$

By induction hypothesis, all the maps specified within brackets above are $Q$ measurable. It follows that $T(d) \in \mathbb{Q}$ for each $d \in S_{j}, \cup\left\{T(d): d \in S_{j}\right\}=T$ and the $T(d)$ 's are pairwise disjoint. Apply Lemma 2.6 with $T(d)$ playing the role of $T$, $V\left(d_{j}\right)-\cup\left\{V\left(d_{i}\right): i<j\right\}$ playing the role of $X$,

$$
G \cap\left(T(d) \times\left(V\left(d_{j}\right)-\cup\left\{V\left(d_{i}\right): i<j\right\}\right)\right)
$$

playing the role of $G$,

$$
G_{k} \cap\left(T(d) \times\left(V\left(d_{j}\right)-\cup\left\{V\left(d_{i}\right): i<j\right\}\right)\right)
$$

playing the role of $G_{0}$, and $\varepsilon=1 /(k+1)$. One obtains $Q$-measurable maps $q_{d}(t, n)$ defined on $T(d)$ satisfying the conditions specified in Lemma 2.6 specialized to the above set-up.

If now one defines $p(t, s n)$ by

$$
p(t, s n)=q_{d}(t, n) \quad \text { on } T(d),
$$

it is easy to verify that all the conclusions of the lemma are satisfied.

We will now drop the assumption on the compactness of $X$ assumed since Lemma 2.4 .

LEMMA 2.8. Let all the assumptions of Lemma 2.7 be in force except the one on the compactness of $X$. Then there is a Caratheodory map $f: T \times \Sigma \rightarrow X$ such that:

(i) $f(t, \cdot)$ is a homeomorphism of $\Sigma$ onto $F(t), t \in T$.

(ii) $f(\cdot, \sigma)$ is an $Q$-measurable selector for $F, \sigma \in \Sigma$.

Proof. By taking a zero-dimensional compactification of $X$ we see that we may assume $X$ to be compact without loss of generality. Let $\{p(t, s): s \in$ Seq $\}$ be then the maps obtained from Lemma 2.7. The result easily follows by defining $f(t, \sigma)$ to be the unique element of $\bigcap_{n \geqslant 1} V(p(t, \sigma \mid n))$. This definition is legitimate and condition (i) is easily seen to be true. Condition (ii) is established by the identity

$$
f(t, \sigma) \in U \leftrightarrow(\exists n)(V(p(t, \sigma \mid n)) \subseteq U)
$$

for each open $U$ in $X$. As $p(t, \sigma \mid n)$ is $Q$-measurable, the right-hand side is an Q-measurable condition.

We will now state the promised uniform version of the Mazurkiewicz theorem.

Theorem 2.1. Let $T$ be Polish, $X$ zero-dimensional and Polish, and $\mathbb{Q}$ a countably generated sub- $\sigma$-field of $\mathscr{B}_{T}$. Let $F: T \rightarrow X$ be an $\mathcal{Q}$-measurable $G_{\delta}$-valued multifunction with $\operatorname{Gr}(F) \in \mathbb{Q} \otimes \mathscr{B}_{X}$. Assume further that for each $t \in T, \operatorname{cl}(F(t))-F(t)$ is dense in $\mathrm{cl}(F(t))$. Then there is a Carathéodory map $f: T \times \Sigma \rightarrow X$ satisfying:

(i) For each $t \in T, f(t, \cdot)$ is a homeomorphism of $\Sigma$ and $F(t)$.

(ii) For each $\sigma \in \Sigma, f(\cdot, \sigma)$ is an Q-measurable selector for $F$.

Proof. Observe that $F(t) \cap U$ is not closed in each clopen set $U$ for which $F(t) \cap U \neq \varnothing$. Lemma 2.8 now applies to yield the theorem. 
Theorem 2.2. Let $T$ be a Polish space and $X$ a zero-dimensional Polish space. Let $Q$

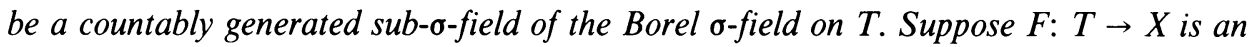
$Q$-measurable multifunction taking dense-in-itself $G_{\AA}$-values. Assume further that $\operatorname{Gr}(F)$ $\in \mathbb{Q} \otimes \mathscr{B}_{X}$. Then there is a Carathéodory map $f: T \times(\Sigma \cup N) \rightarrow X$ such that

(i) for each $t \in T, f(t, \cdot)$ is a 1-1, continuous map of $\Sigma \cup N$ onto $F(t)$ such that $\left.f(t, \cdot)\right|_{\Sigma}$ is a homeomorphism.

(ii) For each $\sigma \in \Sigma, f(\cdot, \sigma)$ is an Q-measurable selector for $F$.

Proof. As $F$ is an $Q$-measurable $G_{\delta}$-valued multifunction, by a theorem of Srivastava [12] it admits a sequence of disjoint $Q$-measurable selectors, say $f_{1}(t)$, $f_{2}(t), \ldots$ such that $\left\{f_{n}(t)\right\}_{n \geqslant 1}$ is dense in $F(t)$ for $t \in T$. (Observe that we can take the $f_{i}$ 's to have disjoint graphs since each $F(t)$ is infinite.) Define a new multifunction $H: T \rightarrow X$ by

$$
H(t)=F(t)-\left\{f_{n}(t)\right\}_{n \geqslant 1} .
$$

Clearly $H(t) \cap U$ is not closed in any clopen set $U$ such that $H(t) \cap U \neq \varnothing$. Notice further that $H$ also satisfies all the remaining conditions required of the multifunction $F$ in Lemma 2.8. This lemma therefore applies to $H$ yielding a suitable Carathéodory representation $h(t, \sigma)$ for $H$. Now put $f(t, \sigma)=h(t, \sigma)$ if $\sigma \in \Sigma$, and $f(t, n)=f_{n}(t)$ if $n \in N$. This $f$ satisfies the conclusions of the theorem.

An easy consequence of Theorem 2.2 is its validity in any Polish space $X$ which becomes zero-dimensional on the removal of countably many points. In particular, Theorem 2.2 holds for multifunctions taking values in $\mathbf{R}$. Thus we have

COROLlary 2.1. Let $T$ be a Polish space and $Q$ a countably generated sub- $\sigma$-field of the Borel $\sigma$-field on $T$. Suppose $F$ is an Q-measurable multifunction on $T \rightarrow \mathbf{R}$ taking dense-in-itself $G_{\delta}$-values and such that, moreover, $\operatorname{Gr}(F) \in Q \otimes \Re_{\mathbf{R}}$. Then the conclusion of Theorem 2.2 holds.

REMARK 2.1. As the maps $f(t, \cdot)$ in Theorem 2.2 are $1-1$, continuous maps, they are, a fortiori, Borel isomorphisms. We have therefore obtained the "Borel parametrization" result of Srivastava and Sarbadhikari [10] for such multifunctions when they take values in (topologically) one-dimensional Polish spaces. However, our proof appears to be more "effective" as it does not go into the Schroder-Bernstein kind of argument employed there. Also, as observed by them, we cannot drop the hypothesis that $F(t)$ is dense-in-itself for each $t$, as then $F$ need not even admit a Borel parametrization.

REMARK 2.2. Let $A$ be an analytic space and $X$ a 0 -dimensional space. Suppose $F$ : $A \rightarrow X$ is a $\mathscr{\bigcap}_{A}$-measurable $G_{\delta}$-valued multifunction such that $\operatorname{Gr}(F) \in \mathscr{B}_{A} \otimes \mathscr{\Re}_{X}$. Fix a Polish space $T$ and a continuous map $f$ on $T$ onto $A$. Put $\mathbb{Q}=f^{-1}\left(\Re_{A}\right)$. Then $\mathbb{Q}$ is a countably generated sub- $\sigma$-field of $\Re_{T}$. Define $H: T \rightarrow X$ by $H(t)=F(f(t))$. Then $H$ is an $Q$-measurable multifunction with $\operatorname{Gr}(F) \in \mathbb{Q} \otimes \mathscr{B}_{X}$. One easily sees now that by assuming various conditions on the values of $F$, which clearly carry over to $H$, one can prove theorems for $\mathscr{\Re}_{A}$-measurable multifunctions on an analytic space $A$, corresponding to all the theorems we have proved so far. The technique is analogous to the one employed in [12]. 
3. One-one Carathéodory representations in a more general setting. In this section we will begin by using the methods developed in the previous section to answer a question posed by Cenzer and Mauldin. In their paper [3], Cenzer and Mauldin have proved the following

THEOREM. Let $W$ be a Borel subset of $I \times I$ such that for each $x, W^{x}$, the vertical section of $W$ at $x$, is uncountable. Then there is a map $h: I \times I$ into $I$ so that

(1) $h$ is an $S(I \times I)$-measurable map (here $S(X)$ denotes the class of $C$-sets in $X$ ),

(2) for each $x, h(x, \cdot)$ is a Borel isomorphism of I onto $W^{x}$,

(3) for each $y, h(\cdot, y)$ is an $S(I)$-measurable selector of $W$, and

(4) if $\hat{h}: I \times I \rightarrow I \times I$ is the canonical map induced by $h$, then $\hat{h}^{-1}$ is $S(I \times I)$ measurable.

However, as is well known, von Neumann [13] and Yankov [16] showed that a Borel subset of $I \times I$ has a $\mathscr{B}(\mathscr{Q}(I))$-measurable selector, where $\mathscr{B}(\mathscr{Q}(I))$ is the $\sigma$-field generated by the analytic subsets of $I$. Cenzer and Mauldin, therefore, asked whether it is possible to so "parametrize" $W$ that the induced individual selectors are $\mathscr{B}(\mathscr{Q}(I))$-measurable. In this section we strengthen the Cenzer-Mauldin theorem so that, on the one hand, the above is accomplished, and on the other, the Borel isomorphisms of Cenzer and Mauldin are replaced by one-one continuous maps. In proving it, we will follow some of the ideas of Ioffe [4]. Later in the section, we will use our methods to prove an abstract parametrization theorem that improves the Cenzer-Mauldin theorem in another direction.

We begin with a few lemmas. The first, which will play a key role in the sequel, employs the methods of the "effective" theory. All notation and results used from the effective theory are from Moschovakis [9], and the reader may refer to it.

Lemma 3.1. Let $P \subseteq \omega^{\omega} \times \omega^{\omega}$ be in $\Delta_{1}^{1}(z)$ for some $z \in \omega^{\omega}$. Then there is a sequence $\left\{f_{n}, n \geqslant 0\right\}$ of $\Pi_{1}^{1}(z)$-recursive partial functions defined on $\omega^{\omega}$ into $\omega^{\omega}$, such that whenever $P^{x}$, the vertical section of $P$ at $x$, is countable,

$$
(\forall y)\left(P(x, y) \leftrightarrow(\exists n)\left(f_{n}(x) \downarrow \text { and } f_{n}(x)=y\right)\right) .
$$

Proof. Fix a $\Pi_{1}^{1}(z)$-recursive partial function $d: \omega^{\omega} \times \omega \rightarrow \omega^{\omega}$ which parametrizes points in $\Delta_{1}^{1}(z, x), x$ running through $\omega^{\omega}$.

Define a predicate $Q \subseteq \omega^{\omega} \times \omega$ as follows:

$$
Q(x, n) \leftrightarrow d(x, n) \downarrow \text { and } P(x, d(x, n)) .
$$

Then $Q$ is in $\Pi_{1}^{1}(z)$. Define, for $n \geqslant 1, f_{n}: \omega^{\omega} \rightarrow \omega$ by

$$
\left(f_{n}(x) \downarrow \text { and } f_{n}(x)=y\right) \leftrightarrow(Q(x, n) \text { and } y=d(x, n)) .
$$

It follows that the $f_{n}$ 's so defined are $\Pi_{1}^{1}(z)$-recursive partial functions. Now, as is well known, any countable $\Delta_{1}^{1}(z, x)$ set contains only $\Delta_{1}^{1}(z, x)$ points. Consequently, for any $x$, if $P^{x}$, a set in $\Delta_{1}^{1}(z, x)$, is countable, we have

$$
(\forall y)(P(x, y) \leftrightarrow(\exists n)(d(x, n) \downarrow \text { and } d(x, n)=y)) .
$$

The conclusion now follows. 
Lemma 3.2. Let $T, X$ be Polish spaces and $A$ a subset of $T$. Suppose $B$ is a Borel subset of $T \times X$ such that $B^{t}$ is countable for each $t \in A$. Then there are countably many partial functions $f_{1}, f_{2}, \ldots$, each defined on a set in $\mathscr{B}(Q(T))$ and measurable with respect to $\Re(Q(T))$, such that

$$
B \cap(A \times X)=\left(\bigcup_{n=1}^{\infty} \operatorname{Gr}\left(f_{n}\right)\right) \cap(A \times X) .
$$

Proof. Without loss of generality, $T$ and $X$ are uncountable and consequently Borel isomorphs of $\omega^{\omega}$. Thus we may assume $B \subseteq \omega^{\omega} \times \omega^{\omega}$. As $B$ is Borel, $B$ is in $\Delta_{1}^{1}(z)$ for some $z$. A straightforward application of Lemma 3.1 together with the facts that the domain of a $\Pi_{1}^{1}(z)$-recursive partial function is a set in $\Pi_{1}^{1}(z)$ and that the inverse image of an open set under a $\Pi_{1}^{1}(z)$-recursive partial function is again a set in $\Pi_{1}^{1}$ yields the lemma.

Lemma 3.3. Let $(T, \Re)$ be a measurable space. Let $F: T \rightarrow \Sigma$ be an T-measurable multifunction taking nonempty and perfect values. Then there is a map $k: T \times(\Sigma \sqcup$ $N) \rightarrow \sum$ such that

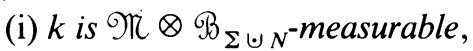

(ii) for each $t \in T, k(t, \cdot)$ is a one-one, continuous map on $\Sigma \cup N$ onto $F(t)$, and

(iii) for each $t \in T$, the restriction of $k(t, \cdot)$ to $\Sigma$ is a homeomorphism.

Proof. As $F$ is a closed-valued measurable multifunction and $\Re$ is a $\sigma$-field, by well-known theorems (see [14]), there are countably many $\mathfrak{N}$-measurable selectors for $F$, say $g_{1}, g_{2}, \ldots$ such that for each $t \in T,\left\{g_{1}(t), g_{2}(t), \ldots\right\}$ is dense in $F(t)$. Moreover, as $F(t)$ is perfect for each $t \in T,\left\{g_{n}(t)\right\}_{n \geqslant 1}$ is a dense-in-itself sequence for every $t \in T$.

We will now reduce the problem to a form to which the methods of $\$ 2$ can readily be applied. To begin with notice that as each $g_{n}$ is $\mathfrak{N}-$ measurable there is a countably generated sub- $\sigma$-field $\mathfrak{R}_{0}$ of $\mathfrak{N}$ such that each $g_{n}$ is measurable with respect to $\mathscr{K}_{0}$. Let $\left\{M_{n}\right\}_{n \geqslant 1}$ be a generator for $\mathscr{K}_{0}$ and let $m: T \rightarrow[0,1]$ be the characteristic function of the sequence $\left\{M_{n}\right\}_{n \geqslant 1}$.

Put $R=m(T)$. The map $m$ is then a bimeasurable map with respect to $\mathfrak{R}_{0}$ and $\Re_{R}$ (that is, a measurable map that carries measurable sets to measurable sets). Thus, to each $g_{n}: T \rightarrow \Sigma$ there corresponds a $\mathscr{B}_{R}$-measurable map $h_{n}: R \rightarrow \Sigma$ such that $g_{n}(t)=h_{n}(m(t)), t \in T$. Hence $F(t)=\operatorname{cl}\left(h_{1}(m(t)), h_{2}(m(t)), \ldots\right)$ for $t \in T$. By (see [6]), each $h_{n}$ extends to a $\mathscr{B}_{[0,1]}$-measurable map $h_{n}^{\prime}:[0,1] \rightarrow \Sigma$.

Let

$$
\begin{aligned}
S & =\left\{s \in[0,1]: h_{n}^{\prime}(s) \text { is a dense-in-itself sequence }\right\} \\
& =\left\{s \in[0,1]:(\forall p \geqslant 1)(\forall n)(\exists k)\left(0<d\left(h_{p}^{\prime}(s), h_{k}^{\prime}(s)\right)<1 /(m+1)\right)\right\},
\end{aligned}
$$

$d$ being a metric on $\Sigma$.

It follows that $S$ is a Borel subset of $[0,1]$. Moreover, as $\left\{g_{n}(t)\right\}_{n \geqslant 1}$ is dense-initself for each $t$, we have

$$
\left\{h_{n}(r)\right\}_{n \geqslant 1}=\left\{h_{n}^{\prime}(r)\right\}_{n \geqslant 1} \text { is dense-in-itself for each } r \in R .
$$

Thus, $R \subseteq S$. 
Define $H: S \rightarrow \Sigma$ by

$$
H(s)=\operatorname{cl}\left(h_{1}^{\prime}(s), h_{2}^{\prime}(s), \ldots\right) .
$$

Then, as is easily checked,

(i) $H$ is a $\mathscr{B}_{S}$-measurable multifunction;

(ii) $\operatorname{Gr}(H) \in \mathscr{B}_{S} \otimes \mathscr{B}_{\Sigma}$;

(iii) $H(s)$ is perfect (and, therefore, a dense-in-itself $G_{\delta}$ ) for each $s \in S$; and

(iv) $H(m(t))=F(t)$ for each $t \in T$.

Thus, as $S$ is absolute Borel, Theorem 2.2 applies to the multifunction $H$ (this is, for example, ensured by Remark 2.2). We therefore obtain a $\mathscr{B}_{S} \otimes \mathscr{B}_{\Sigma \cup N}$-measurable Carathéodory map $k_{0}: S \times(\Sigma \cup N) \rightarrow \Sigma$ satisfying:

(a) $k_{0}(s, \cdot)$ is a one-one, continuous map on $\Sigma \cup N$ onto $H(s)$ for each $s \in S$, and

(b) $k_{0}(s, \cdot)$ restricted to $\Sigma$ is a homeomorphism for each $s \in S$.

Now define $k: T \times(\Sigma \cup N) \rightarrow \Sigma$ by

$$
k(t, \sigma)=k_{0}(m(t), \sigma) .
$$

As $R \subseteq S$, the map $k$ is well defined on the whole of $T$. It is easily seen that this $k$ satisfies the required conditions.

We will now state the first theorem of this section.

THEOREM 3.1. Let $T, X$ be Polish spaces and $W$ a Borel subset of $T \times X$ such that $W^{t}$ is uncountable for each $t \in T$. Then there is a map $h: T \times(\Sigma \cup N) \rightarrow X$ satisfying

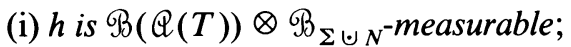

(ii) $h(t, \cdot)$ is a one-one, continuous map on $\Sigma \cup N$ onto $W^{t}$ for each $t \in T$; and

(iii) if $\hat{h}: T \times(\Sigma \cup N) \rightarrow T \times X$ is the canonical map induced by $h$ then $\hat{h}$ is $\left(\mathscr{B}(\mathscr{Q}(T)) \otimes \mathscr{B}_{\Sigma \cup N}, \mathscr{B}_{(}(\mathbb{Q}(T)) \otimes \mathscr{B}_{X}\right)$-measurable and $\hat{h}^{-1}$ is $\left(\left.\mathscr{B}(\mathbb{Q}(T)) \otimes \Re_{X}\right|_{W}\right.$, $\left.\mathscr{B}(Q(T)) \otimes \mathfrak{B}_{\Sigma \cup N}\right)$-measurable.

Proof. Following Cenzer and Mauldin [3], we begin by reducing the problem to one of "parametrizing" a set in $T \times \Sigma$ with uncountable, closed sections. To see this notice that as $W$ is a Borel subset of the Polish space $T \times X$, it is the image under a one-one, continuous map $f$ of a closed subset $C$ of $\Sigma$. Denote by $B$ the set $\left\{(t, \sigma) \in T \times C: \pi_{T}(f(\sigma))=t\right\}$. Then $B$ is a Borel subset of $T \times \Sigma$ with uncountable, closed sections. Moreover, the function $g: B \rightarrow W$ defined by $g(t, \sigma)=f(\sigma)$ is a Borel isomorphism of $B$ onto $W$, and $\pi_{X} \circ f$ maps $B^{t}$ onto $W^{t}$ in a one-one continuous manner. It follows that it suffices to prove our theorem for such subsets $B$ of $T \times \Sigma$.

So let $B \subseteq T \times \Sigma$ be such that $B^{t}$ is closed and uncountable for each $t \in T$. Let $A$ be the perfect kernel of $B$, that is, define $A \subseteq T \times \Sigma$ by

$$
A^{t}=\text { perfect kernel of } B^{t} \text {. }
$$

Then $A$ is an analytic subset of $T \times \Sigma$ with nonempty, perfect sections. Thus $T \times \Sigma-A$ is a coanalytic set with open sections, and we may write

$$
T \times \Sigma-A=\bigcup_{n \geqslant 1}\left(S_{n} \times V_{n}\right),
$$


where $S_{n}$ is coanalytic in $T$ and $V_{n}$ is open in $\Sigma$. Consequently,

$$
B-A=\bigcup_{n \geqslant 1}\left(B \cap\left(S_{n} \times V_{n}\right)\right) \text {. }
$$

Now, $B \cap\left(T \times V_{n}\right)$ is a Borel set having countable sections on $S_{n}$ and Lemma 3.2, therefore, shows that each $B \cap\left(S_{n} \times V_{n}\right)$ is a countable union of graphs of $\mathscr{B}\left(\mathbb{Q}(T)\right.$ )-measurable functions each defined on a set in $\mathscr{B}\left(\mathbb{Q}(T)\right.$ ) (note that each $S_{n}$ being coanalytic is in $\Re(\mathscr{Q}(T))$ ). It follows that $B-A$ is a countable union of graphs of such functions, say, $g_{1}, g_{2}, \ldots$

If we now define a multifunction $F: T \rightarrow \Sigma$ by

$$
F(t)=A^{t}
$$

then $F$ is a nonempty, perfect set-valued multifunction measurable with respect to $\mathscr{B}(Q(T))$. Lemma 3.3 plainly applies to yield a map $k: T \times(\Sigma \cup N) \rightarrow \Sigma$ such that

(a) $k$ is $\Re(Q(T)) \otimes \Re_{\Sigma \cup N}$-measurable;

(b) for each $t \in T, k(t, \cdot)$ is a one-one, continuous map on $\Sigma \cup N$ onto $A^{t}$; and

(c) for each $t \in T$, the restriction of $k(t, \cdot)$ to $\Sigma$ is a homeomorphism.

Put $k_{n}(t)=k(t, n)$ for $n \in N$. Then each $k_{n}$ is a $\mathscr{B}(\mathbb{Q}(T))$-measurable function on $T$ into $\Sigma$. Further, for each $t \in T,\left\{g_{n}(t)\right\}_{n \geqslant 1} \cup\left\{k_{n}(t)\right\}_{n \geqslant 1}$ is infinite (since $\left\{k_{n}(t)\right\}_{n \geqslant 1}$ is so). It follows that there exist $\mathscr{B}(Q(T))$-measurable functions $f_{n}: T \rightarrow \Sigma$ with disjoint graphs such that

$$
\bigcup_{n \geqslant 1} \operatorname{Gr}\left(f_{n}\right)=\left(\bigcup_{n \geqslant 1} \operatorname{Gr}\left(g_{n}\right)\right) \cup\left(\bigcup_{n \geqslant 1} \operatorname{Gr}\left(k_{n}\right)\right) \text {. }
$$

Finally define $h: T \times(\Sigma \cup N) \rightarrow \Sigma$ by

$$
\begin{array}{ll}
h(t, \sigma)=k(t, \sigma) & \text { if } \sigma \in \Sigma, \\
h(t, n)=f_{n}(t) & \text { if } n \in N .
\end{array}
$$

Then, for each $t \in T, h(t, \cdot)$ is a map on $\Sigma \cup N$ onto $B^{t}$. It is easily seen that $h$ satisfies conditions (i) and (ii) specified in the theorem. The measurability of the map $\hat{h}$ specified in (iii) is an easy consequence of the measurability of $h$ and the product structure of the $\sigma$-field sitting on the range of $\hat{h}$. As for the measurability of $\hat{h}^{-1}$ specified in the theorem, a careful look at the construction of the map $h$ shows that this will be established the moment we have shown

Lemma 3.4. Let $(T, \mathfrak{T})$ be a measurable space and let $X$ be Polish. Suppose $f$ :

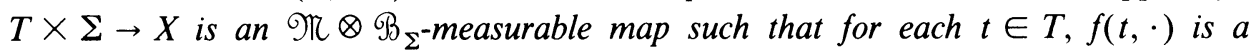
homeomorphism on $\Sigma$ into $X$. Then the canonical map $\hat{f}: T \times \Sigma \rightarrow X$ induced by $f$ satisfies $\hat{f}(M) \in \mathfrak{M} \otimes \Re_{X}$ for each $M \in \mathfrak{K} \otimes \mathscr{B}_{\Sigma}$.

Proof. Plainly, it suffices to show that the range of $\hat{f}$ is in $\mathscr{R} \otimes \Re_{X}$ (for then the image of every basic rectangle would be so).

We begin by observing that for each open $U \subseteq X, f^{-1}(U)$ is a set in $\Re \otimes \Re_{\Sigma}$ with open sections. It is easy to see then that for each $\left(n_{1}, n_{2}, \ldots, n_{k}\right) \in$ Seq, the sets $\left\{t:\left(f^{-1}(U)\right)^{t} \subseteq \Sigma\left(n_{1} n_{2} \cdots n_{k}\right)\right\}$ and $\left\{t:\left(f^{-21}(U)\right)^{t} \neq \varnothing\right\}$ are both in $\Re$.

Fix now for each $k \geqslant 1$, a countable base $\{V(k, m)\}_{m \geqslant 1}$ for $X$, so that for each $m \geqslant 1, \delta(V(k, m))<\frac{1}{k}$. 
Define, for $k \geqslant 1, G_{k} \subseteq T \times X$ by

$$
\begin{aligned}
& (t, x) \in G_{k} \leftrightarrow(\exists m \geqslant 1)\left(\exists\left(n_{1} n_{2}, \ldots, n_{k}\right) \in S_{k}\right) \\
& {\left[x \in V(k, m) \text { and }\left(f^{-1}(V(k, m))\right)^{t} \subseteq \Sigma\left(n_{1} n_{2} \cdots n_{k}\right)\right.} \\
& \left.\quad \text { and }\left(f^{-1}(V(k, m))\right)^{t} \neq \varnothing\right] .
\end{aligned}
$$

Put $G=\bigcap_{k \geqslant 1} G_{k}$. Then $G \in \mathscr{M} \otimes \Re_{X}$.

Claim. Range of $\hat{f}=G$.

Fix $t \in T$. Let $(t, x) \in$ Range of $\hat{f}$. Then by the continuity of the inverse of $f(t, \cdot)$ and the fact that $\{V(k, m)\}_{m \geqslant 1}$ is a base for $X$, we have $(t, x) \in G_{k}$ for each $\dot{k} \geqslant 1$.

Conversely, suppose $(t, x) \in G_{k}$ for every $k \geqslant 1$. Then for each $k \geqslant 1$, we have a natural number $m_{k}$ and $\left(n_{1}^{k}, \ldots, n_{k}^{k}\right) \in S_{k}$ such that $x \in V\left(k, m_{k}\right)$,

$$
f(t, \cdot)^{-1}\left(V\left(k, m_{k}\right)\right) \subseteq \Sigma\left(n_{1}^{k} \ldots n_{k}^{k}\right),
$$

and

$$
f(t, \cdot)^{-1}\left(V\left(k, m_{k}\right)\right) \neq \varnothing \quad \text { for each } k \geqslant 1 .
$$

Consider $m_{1}$ and $\left(n_{1}^{1}\right)$. As $x \in V\left(k, m_{k}\right)$ for every $k \geqslant 1$ and $\delta\left(V\left(k, m_{k}\right)\right)<\frac{1}{k}$, it follows that there is a $k>1$ such that $V\left(k, m_{k}\right) \subseteq V\left(1, m_{1}\right)$. Therefore,

$$
f(t, \cdot)^{-1}\left(V\left(k, m_{k}\right)\right) \subseteq f(t, \cdot)^{-1}\left(V\left(1, m_{1}\right)\right) .
$$

It follows that $\Sigma\left(n_{1}^{k} \cdots n_{k}^{k}\right) \cap \Sigma\left(n_{1}^{1}\right) \neq \varnothing$, and consequently that $\left(n_{1}^{k} \cdots n_{k}^{k}\right)$ is an extension of $\left(n_{1}^{1}\right)$.

Applying the same argument to $m_{k}$ and $\left(n_{1}^{k}, \ldots, n_{k}^{k}\right)$ and so on, we see that there is a single sequence $\left(n_{1}, n_{2}, \ldots\right) \in \Sigma$ and an increasing sequence $\left(k_{1} k_{2}, \ldots\right)$ of positive integers such that

(i) for each $l \geqslant 1,\left(n_{1}, n_{2}, \ldots, n_{k_{l}}\right)=\left(n_{1}^{k_{l}}, \ldots, n_{k_{l}}^{k_{l}}\right)$;

(ii) $\varnothing \neq f(t, \cdot)^{-1}\left(V\left(k_{l}, m_{k_{l}}\right)\right) \subseteq \Sigma\left(n_{1} \cdots n_{k_{l}}\right)$; and

(iii) $x \in V\left(k_{l}, m_{l}\right)$.

Put $y=f\left(t,\left(n_{1}, n_{2}, \ldots\right)\right)$. The continuity of the map $f(t, \cdot)$ now shows that $y=x$ and, therefore, that $(t, x) \in$ Range of $\hat{f}$. This proves the claim, thereby completing the proof of Lemma 3.3 and, consequently, of Theorem 3.1.

REMARK 3.1. As observed at the beginning of this section the von Neumann selection theorem is the best possible selection theorem for an arbitrary Borel set, in the sense that the analytic $\sigma$-field is the smallest natural $\sigma$-field with respect to which one can always get a measurable selection. We are not interested here in the descriptive nature of the graph of the selection. Our Theorem 3.1 therefore cannot be improved in the general setting in which it is stated. Mauldin [7] has obtained interesting necessary and sufficient conditions for a Borel set in the product to be Borel parametrizable. Our Theorem 3.1 together with this result of Mauldin, therefore, seems to be a complete solution to the problem of parametrizing Borel sets in the product of two Polish spaces.

We will now observe that our methods also yield a solution to another kind of parametrization problem that owes its motivation to the following theorem of Ioffe [4] and Bourgain [2]. 
THEOREM. Let $(T, \Re, \mu)$ be a complete measure space, $\mu$ being a $\sigma$-finite measure. Let $X$ be Polish, and suppose $B$ is a subset of $T \times X$ which belongs to $\Re \otimes_{\Re_{X}}$. Assume that $B$ has uncountable vertical sections. Then there is a Carathéodory map $f$ : $T \times(\Sigma \cup N) \rightarrow X$ such that

(i) $f$ is $\mathfrak{R} \otimes \mathscr{B}_{X}$-measurable, and

(ii) for each $t \in T, f(t, \cdot)$ is a 1-1, continuous map of $\Sigma \cup N$ onto $B^{t}$.

Ioffe raised the question whether the above holds when $\mathfrak{T}$ is any $\sigma$-field closed under operation $(\mathbb{Q})$. This our methods easily accomplish as we shall see now. Indeed, we will prove the following abstract theorem which amounts to showing that Theorem 3.1 holds even when $T$ is an arbitrary subset of a Polish space.

If $\mathcal{T}$ is a $\sigma$-field on a set $T$, then $\mathscr{Q}(\mathcal{K})$ will denote the family of all subsets of $T$ obtained as the result of operation $(\mathbb{Q})$ performed on a system of sets from $\mathfrak{N}$.

TheOREM 3.2. Let $(T, \Re)$ be a measurable space. Let $X$ be Polish and suppose $B \in \mathfrak{N} \otimes \mathscr{B}_{X}$ has uncountable vertical sections. Let $\mathscr{R}=\sigma(\mathbb{Q}(\mathfrak{T}))$. Then there is a one-one Carathéodory map $h: T \times(\Sigma \cup N) \rightarrow$ X satisfying

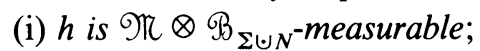

(ii) $h(t, \cdot)$ is a one-one, continuous map of $\Sigma \cup N$ onto $B^{t}$ for each $t \in T$; and

(iii) if $\hat{h}: T \times(\Sigma \cup N) \rightarrow T \times X$ is the canonical map induced by $h$, then for each $M \in \Re \otimes \Re_{\Sigma \cup N}, \hat{h}(M) \in \Re \otimes \Re_{\Sigma \cup N}$.

Proof. As the proof is similar to that of Theorem 3.1, we will only outline the proof. Notice that as $B \in \mathscr{N} \otimes \mathscr{B}_{X}$, there are countably many rectangles $\left\{T_{n} \times V_{n}\right\}_{n \geqslant 1}$, with $T_{n} \in \mathcal{X}$ and $V_{n}$ open in $X$, such that

$$
B \in \sigma \text {-field generated by }\left\{T_{n} \times V_{n}\right\}_{n \geq 1} \text {. }
$$

Let $\mathfrak{R}_{0}=\sigma\left(\left\{T_{n}\right\}_{n \geqslant 1}\right)$ and let $m:\left(T, \Re_{0}\right) \rightarrow[0,1]$ be the characteristic function of the sequence $\left\{T_{n}\right\}$. Put $m(T)=R$ and let $W \subseteq R \times X$ be defined by

$$
W=\{(m(t), x):(t, x) \in B\} .
$$

Then $W \in \mathscr{B}_{R} \otimes \Re_{X}$ and $W^{r}$ is uncountable for each $r \in R$. Also observe that

$$
m^{-1}(\mathscr{B}(\mathscr{Q}(R))) \subseteq \sigma\left(\mathscr{Q}\left(\mathscr{K}_{0}\right)\right) \subseteq \sigma(\mathscr{Q}(\mathscr{K}))=\mathscr{K} .
$$

Easy arguments now show that it is enough to parametrize $W$ by a map $h$ satisfying all the conditions specified in Theorem 3.1 with $T$ replaced by $R$. To see the existence of such a parametrization one has only to follow the various steps in the proof of Theorem 3.1 .

As $W \in \mathscr{B}_{R} \otimes \mathscr{B}_{X}$, there is absolute Borel $W^{\prime} \in \mathscr{B}_{[0,1]} \otimes \mathscr{B}_{X}$ such that $W^{\prime} \cap(R$ $\times X)=W$. Now find $B \subset[0,1] \times \Sigma, B \in \mathscr{B}_{[0,1]} \otimes \Re_{\Sigma}$ with closed vertical sections and a Borel isomorphism $g$ of $B$ onto $W^{\prime}$ as in the proof of Theorem 3.1. Notice that $B^{t}$ is uncountable whenever $\left(W^{\prime}\right)^{t}$ is. Thus, as before, the problem of parametrizing $W^{\prime}$ on $R$ reduces to that of parametrizing $B$ on $R$.

Once again define $A \subseteq[0,1] \times \Sigma$ by

$$
A=\left\{(t, x): x \in \text { perfect kernel of } B^{t}\right\} \text {. }
$$


Arguments identical to ones used in Theorem 3.1 now show that $B-A$ can be written as a countable union of graphs of $\mathscr{B}(\mathscr{Q}([0,1]))$-measurable functions and, consequently, that $(B-A) \cap(R \times \Sigma)$ is a countable union of graphs of $\mathscr{B}(\mathbb{Q}(R))$ measurable functions. On the other hand, $A$ being an analytic set with perfect sections, Lemma 3.3 applies to yield a suitable parametrization of $A$ on $\pi_{[0,1]}(A)$. As $B^{t}$ is uncountable for each $t \in R$, we have $R \subseteq \pi_{[0,1]}(A)$. Thus $A$ and therefore $B$ can be parametrized on $R$. This completes the proof.

The following is now immediate and answers Ioffe's question.

Corollary 3.1. Let $(T, \Re)$ be a measurable space, $M$ being a $\sigma$-field closed under operation $(\mathbb{Q})$. Let $X$ be Polish and suppose $B \in \Re_{\mathbb{Q}} \otimes \mathscr{B}_{X}$ has uncountable vertical sections. Then there is a one-one Carathéodory map $h: T \times(\Sigma \cup N) \rightarrow X$ satisfying (i)-(iii) above.

4. One-one Carathéodory representations for sets with condensed sections. We will now improve some of our earlier results by showing that the dense-in-itself $G_{\delta}$-valued multifunction $F$ in Theorem 2.2 is induced by an $\mathscr{Q} \otimes \mathscr{B}_{\Sigma}$-measurable one-one Carathéodory map defined on $T \times \Sigma$ and also that if in Corollary 3.1 it is assumed that the set $B$ has condensed sections, then $B$ is induced by a one-one Carathéodory map defined on $T \times \Sigma$ with all the measurability properties specified in Corollary 3.1. These answer questions raised in [14]. We will follow some of the ideas in Sierpinski's proof that a condensed Borel set in $R$ is a one-one continuous image of $\Sigma[11]$

We begin with several lemmas. In what follows $X$ denotes a fixed Polish space. Suppose $T$ is an abstract set and $\Re$ a $\sigma$-field on $T$. Let $h: T \times \Sigma \rightarrow X$ be a $\mathfrak{T} \otimes \mathscr{B}_{\Sigma}$-measurable, one-one, Carathéodory map and $s_{0}: T \rightarrow X$ an $\mathfrak{T}$-measurable map such that

(i) $s_{0}(t) \notin \operatorname{Range}(h(t, \cdot))$ for $t \in T$, and

(ii) $s_{0}(t) \in \operatorname{cl}(\operatorname{Range}(h(t, \cdot)))$ for $t \in T$.

Put $C=\{(t, x) \in T \times X:(\exists \sigma \in \Sigma)(h(t, \sigma)=x)\}$ and $B=C \cup \operatorname{Gr}\left(s_{0}\right)$. Finally, let $d$ be a fixed metric on $X$.

Lemma 4.1. Suppose $r: T \rightarrow X$ is $\Re$-measurable. Then $\{(t, x): d(x, r(t))<\varepsilon\}$ is a set in $\Re \otimes \mathscr{B}_{X}$ with nonempty and open vertical sections for each $\varepsilon>0$.

Proof. This is clear.

Lemma 4.2. Suppose $P \subseteq T \times \Sigma, P \in \mathfrak{R} \otimes \mathscr{B}_{\Sigma}$, and $P^{t}$ is nonempty and open for each $t \in T$. Assume that for each open $U \subseteq \Sigma,\left\{t \in T: U \subseteq P^{t}\right\} \in \mathfrak{N}$. Then there is a map $q: T \times \Sigma \rightarrow \Sigma$ such that

(i) $q$ is $\Re \otimes \mathscr{B}_{\Sigma^{-}}$measurable, and

(ii) for each $t \in T, q(t, \cdot)$ is a homeomorphism on $\Sigma$ onto $P^{t}$.

Proof. Fix an enumeration $u_{1}, u_{2}, \ldots$ of Seq. Define

$$
T_{n}=\left\{t \in T: \Sigma\left(u_{n}\right) \subseteq P^{t} \text { and } \Sigma(s) \varsubsetneqq P^{t} \text { for every } s \prec u_{n}\right\} .
$$

Then $T_{n} \in \Re$ (by our assumptions on $\Re$ ). 
Define $\Re$-measurable maps $p(t, n), n \geqslant 1$, taking values in Seq $U\{\infty\}$ as follows (we put $\Sigma(\infty)=\varnothing)$ :

$$
p(t, 1)=u_{m} \quad \text { if } m \text { is the first integer } l \text { such that } t \in T_{l}
$$

and for $n \geqslant 1$,

$$
\begin{array}{ll}
p(t, n+1)=u_{m} & \begin{array}{l}
\text { if } m \text { is the first integer } l>p(t, n) \\
\text { such that } t \in T_{l}, \text { if there is one such; }
\end{array} \\
=\infty & \text { otherwise. }
\end{array}
$$

As each $T_{l} \in \mathscr{N}$, the maps $p(t, n)$ are $\mathfrak{N}$-measurable. Further, they satisfy

(a) $\{\Sigma(p(t, n)): n \geqslant 1\}$ is a discrete family in the topology relativised to their union; and

(b) $P^{t}=\cup_{n \geqslant 1} \Sigma(p(t, n)), t \in T$.

Let

$$
\begin{gathered}
S_{0}=\{t \in T: p(t, n) \neq \infty \text { for every } n \geqslant 1\}, \\
S_{n}=\{t \in T: p(t, n) \neq \infty \text { and } p(t, n+1)=\infty\}, \quad n \geqslant 1 .
\end{gathered}
$$

Notice that $\cup_{n \geqslant 0} S_{n}=T$, the $S_{n}$ 's are disjoint, and $S_{n} \in \Re, n \geqslant 0$. For each $s$, $t \in$ Seq, fix a homeomorphism of $\Sigma(s)$ onto $\Sigma(t)$, say $h(s, t)$.

Define $f_{0}: S_{0} \times \Sigma \rightarrow \Sigma$ by

$$
f_{0}(t, \sigma)=h(\langle k\rangle, p(t, k))(\sigma) \text { if } \sigma \in \Sigma(\langle k\rangle) .
$$

As the $p(t, n)$ 's are M-measurable, $f_{0}$ has the following properties:

(1) $f_{0}(t, \cdot)$ is a homeomorphism of $\Sigma$ onto $P^{t}$ for $t \in S_{0}$;

(2) $f_{0}$ is $\left.\Re\right|_{S_{0}} \otimes \Re_{\Sigma}$-measurable.

As any finite disjoint union of basic clopen sets $\Sigma(s)$ in $\Sigma$ is a homeomorph of $\Sigma$, for each $n \geqslant 1$, we can similarly construct maps $f_{n}: S_{n} \times \Sigma \rightarrow \Sigma$ with the above properties. Piecing together these maps we obtain a map $q$ with the required properties.

REMARK 4.1. If in Lemma 4.2 we assume that each $P^{t}$ is clopen, then the condition $\left\{t: U \subset P^{t}\right\} \in \mathscr{T}$ holds if for $\Re$ we take a countably generated sub- $\sigma$-field of the Borel $\sigma$-field on a Polish space. The conclusion of the lemma consequently holds in this situation. This will be used in the sequel.

Lemma 4.3. Suppose $P \subseteq T \times \Sigma$ is in $\Re \otimes \Re_{\Sigma}$ and has nonempty, open sections. Then $P$ has an T-measurable selector.

Proof. Fix a countable dense set $\left\{d_{n}\right\}_{n \geqslant 1}$ in $\Sigma$. Put $T_{n}=\left\{t:\left(t, d_{n}\right) \in P\right\}$. Then $T_{n} \in \Re, n \geqslant 1$, and $\cup_{n \geqslant 1} T_{n}=T$ as each $P^{t}$ is open and nonempty. As $\Re$ is a $\sigma$-field there is a sequence $S_{n}$ such that $S_{n} \subseteq T_{n}, \cup_{n \geqslant 1} S_{n}=\cup_{n \geqslant 1} T_{n}$, and the $S_{n}$ 's are disjoint. Set $f=d_{n}$ on $S_{n}, n \geqslant 1$. Then $f$ is a $\Re$-measurable selector for $P$.

LEMMA 4.4. There is a sequence of M-measurable functions $s_{n}: T \rightarrow X, n \geqslant 1$, such that

(i) $s_{n}(t) \in C^{t}$, for $t \in T$ and $n \geqslant 1$, and

(ii) $d\left(s_{n+1}(t), s_{0}(t)\right)<\frac{1}{3} \cdot d\left(s_{n}(t), s_{0}(t)\right), n \geqslant 1$. 
Proof. Fix $\sigma \in \Sigma$ and define $s_{1}: T \rightarrow X$ by $s_{1}(t)=h(t, \sigma)$. Suppose $s_{n}$ has been defined. Then consider

$$
P=\left\{(t, x): d\left(x, s_{0}(t)\right)<\frac{1}{3} \cdot d\left(s_{n}(t), s_{0}(t)\right)\right\} .
$$

As $s_{0}$ and $s_{n}$ are $\mathfrak{N}$-measurable, it follows from Lemma 4.1 that $P \in \mathfrak{R} \otimes \mathscr{B}_{X}$ and $P$ has open sections. Now put $Q=\left\{(t, \sigma) \in T \times \Sigma: h(t, \sigma) \in P^{t}\right\}$. Then $Q \in \Re \otimes$ $\mathscr{B}_{\Sigma}$. Also $Q$ has open sections as $h(t, \cdot)$ is continuous for each $t \in T$, and, further, since $s_{0}(t)$ is a limit point of $C^{t}$ for each $t, Q^{t}$ is nonempty for each $t \in T$. By Lemma 4.3 there is a measurable selector $q: T \rightarrow X$ for $Q$. Put $s_{n+1}(t)=h(t, q(t))$. This has the required properties.

LEMMA 4.5. Let $T$ be Polish (respectively an abstract set) and $\mathfrak{T}$ a countably generated sub- $\sigma$-field of $\Re_{T}$ (respectively a $\sigma$-field on $T$ closed under operation $(\mathbb{Q})$ ). Suppose $P \subseteq T \times \Sigma, P \in \mathfrak{N} \otimes \mathfrak{B}_{\Sigma}$, and $P^{t}$ is nonempty and open for each $t \in T$. Then there is $Q \subseteq P$ such that $Q \in \Re \otimes \Re_{\Sigma}$, and $Q^{t}$ is nonempty and clopen for each $t \in T$.

Proof. We will prove the lemma in the case when $T$ is Polish. The other case is even simpler. Put $T_{s}=\left\{t \in T: \Sigma(s) \subseteq P^{t}\right\}$ for $s \in$ Seq. Then $T_{s}$ is a coanalytic set and $\cup_{s \in \text { Seq }} T_{s}=T$. Further, $T_{s}$ is a union of atoms of $\Re$. By an invariant reduction principle (see [12]), there exist sets $R_{s} \in \mathfrak{T}, s \in$ Seq, such that $R_{s} \cap R_{s^{\prime}}=\varnothing$ for $s \neq s^{\prime}, R_{s} \subset T_{s}$ and $\cup_{s \in \text { Seq }} R_{s}=\cup_{s \in \text { Seq }} T_{s}$. Now define $Q=\cup_{s \in \text { Seq }} R_{s} \times \Sigma(s)$. This does the job.

LEMMA 4.6. Let the assumptions of Lemma 4.5 be in force. Then there is a countable family $\left\{U_{n}\right\}_{n \geqslant 1}$ of subsets of $T \times \Sigma$ in $\Re \otimes \Re_{\Sigma}$ satisfying

(i) $U_{n}^{t}$ is nonempty and clopen for each $t \in T$;

(ii) the family $\left\{U_{n}^{t}\right\}_{n \geqslant 1}$ is discrete in $\Sigma$, and consequently $\cup_{n \geqslant 1} U_{n}^{t}$ is closed in $\Sigma$ for each $t \in T$;

(iii) $\Sigma-\cup_{n \geqslant 1} U_{n}^{t}$ is nonempty for each $t \in T$; and

(iv) $d\left(h(t, \cdot)\left(U_{n}^{t}\right), s_{0}(t)\right) \downarrow 0$ as $n \rightarrow \infty$.

Proof. As $C$ is induced by the map $h$ easy arguments show that there is a set $S_{0} \subset T \times X$ such that $S_{0} \in \mathscr{N} \otimes \mathscr{B}_{X}, S_{0}^{t}$ is open, $s_{0}(t) \in S_{0}^{t}$, and $C^{t}-S_{0}^{t} \neq \varnothing$ for each $t \in T$. Now find $s_{n}: T \rightarrow X$ as in Lemma 4.4 such that, further, $s_{n}(t) \in S_{0}^{t}$ for each $n \geqslant 1$. This can easily be done. Now put, for $n \geqslant 1$,

$$
V_{n}=\left\{(t, x) \in T \times X: d\left(x, s_{n}(t)\right)<\frac{1}{3} \cdot d\left(s_{n}(t), s_{0}(t)\right) \text { and }(t, x) \in S_{0}\right\} .
$$

Then $V_{n} \in \mathfrak{N} \otimes \mathscr{B}_{X}$ and for each $t \in T, V_{n}^{t}$ is open, $C^{t}-V_{n}^{t} \neq \varnothing,\left\{V_{n}^{t}\right\}_{n \geqslant 1}$ is a family discrete in $C^{t}$, and $d\left(V_{n}^{t}, s_{0}(t)\right) \rightarrow 0$ as $n \rightarrow \infty$.

Define $W_{n} \subseteq T \times \Sigma$ by

$$
W_{n}=\left\{(t, \sigma) \in T \times \Sigma: h(t, \sigma) \in V_{n}^{t}\right\} .
$$

Then, for $n \geqslant 1, W_{n} \in \mathscr{N} \otimes \mathscr{R}_{\Sigma}$ and $W_{n}^{t}$ is open and nonempty for each $t \in T$. Apply Lemma 4.5 to obtain $U_{n} \in \Re_{R} \otimes \mathscr{B}_{\Sigma}$ such that $U_{n}^{t}$ is nonempty and clopen for each $t \in T$, and $U_{n} \subseteq W_{n}$. As the inverse image under a continuous map of a family 
discrete in the range of the map is discrete in the domain, one easily verifies that the family $\left\{U_{n}\right\}_{n \geqslant 1}$ obtained above satisfies conditions (i)-(iv). This completes the proof of the lemma.

We are now in a position to prove the first theorem of this section.

TheOREM 4.1. Let $T$ be Polish and $Q$ a countably generated sub-o-field of the Borel $\sigma$-field on $T$. Let $F: T \rightarrow R$ be an Q-measurable multifunction taking dense-in-itself $G_{\delta}$-values and further satisfying $\operatorname{Gr}(F) \in \mathbb{Q} \otimes \Re_{R}$. Then there is a map $f: T \times \Sigma \rightarrow R$ such that

(i) $f(t, \cdot)$ is a one-one, continuous map on $\Sigma$ onto $F(t)$ for $t \in T$, and

(ii) $f(\cdot, \sigma)$ is an Q-measurable selector for $F$.

Proof. As $F$ satisfies all the hypotheses of Theorem 2.2, there is (in view of Remark 2.1) a map $h: T \times(\Sigma \cup N) \rightarrow R$ such that

(a) $h(t, \cdot)$ is a one-one, continuous map on $\Sigma \cup N$ onto $F(t)$ for each $t \in T$, and

(b) $h(\cdot, \sigma)$ is an $Q$-measurable selector for $F$ for each $\sigma \in \Sigma \biguplus N$.

One can get countably many disjoint condensed Borel sets $C_{n}, n \geqslant 1$, each dense in $\Sigma$ such that $\cup_{n \geqslant 1} C_{n}=\Sigma$ (see [11]). Each $C_{n}$, being condensed Borel, is a one-one, continuous image of $\Sigma([11])$. Thus, as is apparent, for each $n \geqslant 1$, there is a one-one Carathéodory map $h_{n}: T \times \Sigma(\langle n\rangle) \rightarrow R$ inducing $\operatorname{Gr}(F) \cap \hat{h}\left(T \times C_{n}\right)=\operatorname{Gr}\left(F_{n}\right)$, say, where $\hat{h}$ is the canonical map induced by $h$. Let $g_{n}: T \rightarrow R$ be defined by $g_{n}(t)=h(t, n)$, for $n \geqslant 1$. Then, as each $F(t)$ is dense-in-itself, it is easy to see that $g_{n}(t)$ is a limit point of $F(t)$, and consequently of $F_{n}(t)$, for each $t \in T$.

Observe that to prove the theorem it suffices to prove that there is a one-one Carathéodory map $f_{n}: T \times \Sigma(\langle n\rangle) \rightarrow R$ inducing $F_{n} \cup \operatorname{Gr}\left(g_{n}\right)$, for then we could define $f: T \times \Sigma \rightarrow R$ by

$$
f(t, \sigma)=f_{n}(t, \sigma) \quad \text { if } \sigma \in \Sigma(\langle n\rangle) .
$$

We will now construct the map $f_{n}$. Notice that without loss of generality we may assume that $h_{n}: T \times \Sigma \rightarrow R$. We will then define $f_{n}: T \times \Sigma \rightarrow R$. Take $h_{n}$ to be the map $h$ fixed in the beginning of this section, $Q$ to be the $\sigma$-field $\Re$ on $T$, and $g_{n}$ to be the map $s_{0}: T \rightarrow R$. Now Lemma 4.6 applies to yield a family $\left\{U_{m}\right\}_{m \geqslant 1}$ satisfying the conditions therein.

Define $U_{0} \subseteq T \times \Sigma$ by $U_{0}^{t}=\Sigma-\cup_{m \geqslant 1} U_{m}^{t}$. Then $U_{0} \in Q \otimes \mathscr{B}_{\Sigma}$ and $U_{0}^{t}$ is clopen and nonempty for each $t \in T$. Also recall $U_{m}^{t}$ is clopen for each $t$ and $m \geqslant 1$.

By Remark 4.1, for each $m \geqslant 0$, there is an $Q \otimes \mathscr{B}_{\Sigma}$-measurable map $p_{m}: T \times \Sigma \rightarrow$ $\Sigma$ such that $p_{m}(t, \cdot)$ is a homeomorphism on $\Sigma$ onto $U_{m}^{t}$ for each $t$.

As $\Sigma$ is a homeomorph of the space $A$ of irrationals in $(-1,1)$ together with $\{0\}$, it is enough to define $f_{n}: T \times A \rightarrow R$, where $A=$ irrationals in $(-1,1) \cup\{0\}$. Fix $\left\{r_{m}\right\}_{m \geqslant 1}$ and $\left\{t_{m}\right\}_{m \geqslant 1}$, both sequences of rationals such that $r_{m} \uparrow 0$ and $t_{m} \downarrow 0$. Take $r_{1}=-1$ and $t_{1}=1$. Put

$$
\begin{aligned}
A_{2 m} & =\left(r_{m}, r_{m+1}\right) \cap A, & & m \geqslant 1, \\
A_{2 m+1} & =\left(t_{m+1}, t_{m}\right) \cap A, & & m \geqslant 1 .
\end{aligned}
$$

Each $A_{m}$ is a homeomorph of $\Sigma$ and we may therefore look upon the maps $p_{l}$ obtained above as defined on any $T \times A_{m} \rightarrow \Sigma$. 
Define $f_{n}: T \times A \rightarrow R$ by cases as follows:

$$
\begin{aligned}
f_{n}(t, \sigma) & =h_{m}\left(t, p_{m-2}(t, \sigma)\right), \quad \text { if } \sigma \in A_{m}, \quad m \geqslant 2 . \\
f_{n}(t, 0) & =g_{n}(t) .
\end{aligned}
$$

The map $f_{n}$ has the required properties. The theorem follows immediately in view of the remarks made earlier.

Remark 4.2. As any zero-dimensional Polish space can be embedded as a $G_{\delta}$ in $R$, Theorem 4.1 implies that the analogous result holds in any zero-dimensional space. However, our method does not go through for a general Polish space. The difficulty lies in our use of Theorem 2.2 and not in the methods developed in this section. We add that R. D. Mauldin and H. Sarbadhikari have independently obtained Theorem $4.1[8]$.

We will now improve Corollary 3.1.

THEOREM 4.2. Let $(T, \Re)$ be a measurable space where $\mathfrak{N}$ is a $\sigma$-field closed under operation $(\mathbb{Q})$. Let $X$ be Polish. Suppose $B \in \mathfrak{R} \otimes \Re_{X}$ has condensed sections. Then there is a map $f: T \times \Sigma \rightarrow X$ satisfying:

(i) $f$ is $\Re \otimes \mathfrak{B}_{\Sigma^{-} \text {-measurable; }}$

(ii) $f(t, \cdot)$ is a one-one, continuous map on $\Sigma$ onto $B^{t}$ for each $t \in T$; and

(iii) if $\hat{f}$ : $T \times \Sigma \rightarrow T \times X$ is the canonical map induced by $f$; then

(a) $\hat{f}$ is $\left(\mathfrak{T} \otimes \mathscr{B}_{\Sigma}, \mathfrak{T} \otimes \mathscr{B}_{X}\right)$-measurable;

(b) $\hat{f}^{-1}$ is $\left(\left.\mathfrak{N} \otimes \mathscr{B}_{X}\right|_{B}, \mathfrak{N} \otimes \mathscr{B}_{\Sigma}\right)$-measurable.

Plainly, any f satisfying the above induces only $B \in \Re \otimes \Re_{X}$ with condensed sections.

Proof. As Lemmas 4.1-4.6 hold in this set-up also, arguments identical to the ones used in the proof of Theorem 4.1 show the existence of a map $f: T \times \Sigma \rightarrow X$ satisfying conditions (i) and (ii). It suffices to verify (iii)(b). By the construction of the map in Theorem 4.1 it follows that it is enough to see that $\left.f_{n}\right|_{T \times A_{m}}$ satisfies this condition for each $m$. By the definition of the map $f_{n}$ it follows that this will be established the moment each $p_{n}$ constructed in the proof satisfies this condition (note that now we have by Corollary 3.1 a map $h: T \times(\Sigma \cup N) \rightarrow X$ inducing $B$ and satisfying condition (iii)(b)). But the validity of this property for $p_{n}$ is immediate from Lemma 3.4. This completes the proof of Theorem 4.2.

\section{REFERENCES}

1. D. Blackwell, On a class of probability spaces, Proc. 3rd Berkeley Sympos. Math. Statist. and Prob., Vol. 2, Univ. of California Press, Berkeley, Calif., 1956, pp. 1-6.

2. J. Bourgain, Ph.D. Thesis, Univ. of Brussels, (1977).

3. D. Cenzer and R. D. Mauldin, Measurable parametrizations and selections, Trans. Amer. Math. Soc. 245 (1978), 399-408.

4. A. D. Ioffe, One-one Carathéodory representation theorem for multifunctions with uncountable values, Fund. Math. 109 (1980), 19-29.

5. K. Kuratowski and A. Mostowski, Set theory, Studies in Logic and the Foundations of Mathematics, Vol. 86, North-Holland, Amsterdam, 1976, pp. 471-472.

6. K. Kuratowski, Topology, Vol. 1, 5th ed., PWN, Warsaw; Academic Press, New York; "Mir", Moscow, 1966. MR 36 \# \#39, 840.

7. R. D. Mauldin, Borel parametrizations, Trans. Amer. Math. Soc. 250 (1979), 223-234. 
8. R. Daniel Mauldin and H. Sarbadhikari, Continuous one-to-one parametrizations, Bull. Soc. Math. France (to appear).

9. Y. N. Moschovakis, Descriptive set theory, Studies in Logic and the Foundations of Mathematics, Vol. 100, North-Holland, Amsterdam, 1980.

10. H. Sarbadhikari and S. M. Srivastava, Parametrization of $G_{\delta^{-v}}$ alued multifunctions, Trans. Amer. Math. Soc. 258 (1980), 457-466.

11. W. Sierpinski, Sur les images biunivoques et continues de l'ensembles de tous les nombres irrationnels, Mathematica 2 (1924), 18.

12. S. M. Srivastava, Selection theorems for $G_{\delta^{-v}}$ alued multifunctions, Trans. Amer. Math. Soc. 254 (1979), 283-293.

13. J. von Neumann, On rings of operators; reduction theory, Ann. of Math. (2) 30 (1949), 401-485.

14. D. H. Wagner, Survey of measurable selection theorems: an update, Proc. Conf. on Measure Theory (Oberwolfach, 1979), Springer-Verlag, Berlin and New York, 1980, pp. 176-219.

15. E. Wesley, Extensions of the measurable choice theorem by means of forcing, Israel J. Math. 14 (1973), $104-114$.

16. W. Yankov, Sur l'uniformisation des ensembles A, Dokl. Akad. Nauk SSSR 30 (1941), 597-598.

Division of Mathematics and Theoretical Statistics, Indian Statistical Institute, 203 B. T. Road, Calcutta 700 035, India 